\title{
A Comparison Between 0,25\% Tumeric Extract Cream and Grated Tumeric for Skin Discoloration
}

\author{
Solecha Setiawati $^{1,}$, , Faridha Ilyas ${ }^{1}$, Farida Tabri ${ }^{1}$, Khairuddin Djawad ${ }^{1}$, Gemini Alam $^{2}$ \\ ${ }^{1}$ Department of Dermatology and Venereology, Faculty of Medicine, Hasanuddin University, Makassar, Indonesian \\ ${ }^{2}$ Department of Pharmacy, Faculty of Pharmacy, Hasanuddin University, Makassar, Indonesian
}

\section{Email address:}

dr.ikasolecha@gmail.com (S. Setiawati)

${ }^{*}$ Corresponding author

\section{To cite this article:}

Solecha Setiawati, Faridha Ilyas, Farida Tabri, Khairuddin Djawad, Gemini Alam. A Comparison Between 0,25\% Tumeric Extract Cream and Grated Tumeric for Skin Discoloration. American Journal of Clinical and Experimental Medicine. Vol. 5, No. 2, 2017 , pp. $50-55$. doi: 10.11648/j.ajcem.20170502.15

Received: January 26, 2017; Accepted: February 10, 2017; Published: March 2, 2017

\begin{abstract}
Turmeric has been found to have some efficacies in repairing the skin texture including its ability to enlighten the color of the skin associated to premature skin aging. This study aims to compare between the $0.25 \%$ turmeric extract cream and grated turmeric in skin color change. Samples in this study were collected from women subjects that were selected randomly and consecutively. There were 22 women subjects involved in this study that were assigned into two treatment groups: group I with one application a day and group II with two applications a day. Each subject in both groups applied $0.25 \%$ turmeric extract cream in upper right arm, control (4\% Hydroquinone) in lower right and left arms, and grated turmeric in upper left arm. The treatment responses were evaluated by skin analyzer measuring the pigment number at day 0,8 , and 21 . Data were analyzed statistically by Willcoxon test. Study findings indicated significant differences $(p<0,05)$ between twice application of $0.25 \%$ turmeric cream extract and $4 \% \mathrm{Hq}$ at day 8 (2.30) on skin changes (pigment). There was also a significant difference $(\mathrm{p}<0,05)$ when comparing the 0,25 turmeric extract in twice application a day between $\mathrm{H} 21-\mathrm{H} 0$ and $\mathrm{H} 21$ H8.
\end{abstract}

Keywords: 0,25\% Turmeric Extract, Hidroquinon 4\%, Skin Discoloration

\section{Introduction}

Skin is a complex organ that provides protection against the external environment and at the same time interacts with its environment. The skin protects body from infectious agent, plays role in thermoregulation, sensation, ultraviolet light protection, wound reparation and regeneration, physical appearance, provitamin D storage and vitamin D formation, functions as an excretion organ through sweat, sensing organ, and in collagen formation. Beneficial effects of plant extracts have been known since the prehistoric times. Modern cosmetology is currently more than ever interested to apply valuable raw plant materials in manufacturing cosmetics products since the plant extracts contain a plethora of active ingredients. Potential skin caring effects are related to their antioxidant and antiinflammatory properties. Exposure of the skin on ultraviolet radiation gives rise to formation of free radical species that react with DNA, proteins and fatty acids leading to various oxidation products. The latter liberate proinflammatory mediators responsible for irritation of the epidermis. Free radicals generated by UV radiation also take part in the process that may damage skin regulatory mechanisms, resulting in visible photoageing effects such as wrinkles, hiperpigmentation and loss of skin firmness. Making an attempt to reduce negative effects of ultraviolet radiation, some raw materials of plant origin are added to cosmetic products in order to incorporate their principal ingredients having potentially protecting and colouring activity, and therefore providing better skin colour similar to natural suntan without the above-mentioned side effects. This activity possesses curcumin (diferuloylmethane), a yellow pigment derived from the rhizome of plant Curcuma longa 
(turmeric). It is a hydrophobic polyphenol active ingredients, dimeric derivative of ferulic acid. Diferuloylmethane belongs to the group of curcuminoids. Turmeric constituents also include the three other curcuminoids: Desmethoxycurcumin, bidesmethoxycurcumin and tetrahydroxycumarin, but only diferuloylmethane is responsible for its characteristic yellow colour. The content of curcumin in rhizome is in range from 3 to $5.4 \%$ and depends on the place of plant orgin. It has been known for a long time that curcuminoids compounds exhibit a broad spectrum of therapeutic activity. The most important are antioxidant, antiinflammatory, antimicrobial and anticarcinogenic activity. Turmeric is also used as natural coloring agent and a spice for food, as well as in pharmaceutical industry. As for a cosmetic purpose, curcumin is used as an active compound in skin care preparations because its antioxidant, antiinflammatory and antiaging activity. Since ages, the paste of turmeric powder has been used for treatment of inflammation and skin injury and also as an antiseptic mainly due to high curcumin content. Curcumin has also been traditionally used for cosmetic purposes as skin colourant [1]. [2]

The skin color is determined by the pigment composition and blood circulation in skin tissues. Generally, the epidermis comprised of two pigments: carotene (pheumelanin) and brown/black pigment (eumelanin). Carotene is an orange yellow pigment accumulated in epidermis layer and shown in the stratum corneum of people with light skin, whereas the eumelanin is a brown, brownish yellow or black pigment produced by melanosis. Melanin pigment functions to protect skin from ultraviolet radiation (UV) of sunlight. [3]

The skin is also a marker of body system alteration in general, for example the aging process that occur in each skin organ will provide the very first signs. The aging process that occurs faster than normal is called premature aging. Many people begin to show wrinkles in their facial skin at relatively young age, even at the beginning of 20s [3]

Free radicals are opportunistic superoxygen molecule that circulate in blood flow and always ready to damage weak or compromised cells. [2] As a part of the cleansing cascade in the body, the free radicals naturally play a great role in tissue remodeling process, and these cells can be activated very easily. Cigarette, motor vehicle smoke and sun light are some the factors that can induce free radicals [4]

Turmeric or Curcuma longa Linn (synonym Curcuma domestica $\mathrm{Val}$ ) from the family of zingiberaceae is of the important medicinal plants with widespread uses in daily life in Indonesia. Turmeric is used in various fields such as health, culinary, and cosmetics. In traditional medicine, turmeric is used as anti-inflammation, antiseptic, antiirritation and anorexia [5].

The most common utilized part of the turmeric is its rhizome for traditional medicines, seasoning, food ingredients, and cosmetic material. This curcuma contains curcuminoid, consisting of curcumin compound and its derivatives that include desmetoxy-curcumin and bisdesmetoxy-curcumin. Curcuminoid is an active substance in turmeric rhizome with wide spectrum of biological activities, including its anti-inflammatory effect [5]. Currently, curcumin is also used in cosmetics as antiacne, anti-inflammation, anti-oxidant, antibacterial, anticancer, anti-cholesterol and anti-platelet aggregation [6].

The main compound, curcumin, is a potent inhibitor of protein kinase C, EGF-receptor tyrosine kinase and IkappaB kinase, and is an effective antioxidant "bioprotectant". [6]

A previous study by Majeed et al (2010) suggests that $0.25 \%$ turmeric extract is an effective skin enhancement agent with multifunction topical benefits. This extract is safe for topical use without irritation or sensitization as side effects. Ingested curcumin will be metabolized into tetrahydrocurcumin (THC) in vivo. Therefore, the tetrahydrocurcumin is the product of curcumin natural biotransformation.

Tyrosinase inhibitor and other agents affecting the melanin biosynthesis pathway are distributed widely in plant materials. These are safer alternative natural active substances for hydroquinon, to be used as topical skin enhancer composition, and may offer additional functionality, as sunscreen, humidifer, or "anti-aging agents". One of these materials derives from spices and turmeric.

When the natural yellow curcuminoid (bisdemethoxycurcumin) from Curcuma longa (turmeric) root is dehydrogenized, the free mixture color of Tetrahydrocurcuminoid is obtained. This natural mixture is considered as topical antioxidant and anti-inflammatory agent, with advantage of free radical scaveging and peroxide lipid inhibition compared to vitamin E. Study suggests that Tetrahydrocurcuminoid, Tetrahydrocurcumin, particularly the ultra pure, are efficient in inhibiting tyrosinase [6]

An in vitro study indicated that this natural substance is an effective "biopectant" antioxidant which is efficient in preventing the formation of free radicals. This substance is also efficient in inhibiting tyrosinase, and more effective compared to kojic acid, 40\% Glabridin (licorice root extract), and vitamin $\mathrm{C}$ (ascorbic acid) used as natural depigmentation agent [7], [8].

Based on the background above, this study was aimed to compare between $0.25 \%$ turmeric extract cream and grated turmeric against the skin discoloration.

\section{Material and Method}

\subsection{Study Location and Time}

This study was conducted in General Hospital of Dr. Wahidin Sudirohusodo Makassar, Education Hospital of Hasanuddin University, and other networked hospitals as sampling locations. The study was conducted for 21 days until the minimum sample number was met.

\subsection{Study Design and Variables}

This study was an analytical observational study with prospective cohort method. Study variables consisted of: independent variables $0.25 \%$ turmeric extract cream is an extract form consist of $0,25 \%$ turmeric, grated turmeric is 
pure turmeric without extraction process but grated and directly applied to the skin, $4 \%$ hydroquinone is the most often ingredient use to make skin fairer, dependent variable (dull skin), intermediate variable (inhibiting tyrosinase enzyme), and moderator variable (stress, UV light, diet, trauma, smoking, free radicals).

\subsection{Population and Sample}

The population of this study was 30-50 years old women presenting to dermatovenereology clinic of Dr. Wahidin Sudirohusodo hospital, Hasanuddin University teaching hospital and other networked hospitals. Study samples were the entire reachable population fulfilling the inclusion criteria. Samples were selected consecutively where all patients presenting to dermatovenereology clinic and fulfilling inclusion criteria were involved to study during specified time until the needed volume of sample was met. Informed consent from the patients and parents for trial samples were taken before the data collected, and have been approved from the biomedic ethical committee for human research, Medical Faculty of Hasanuddin University.

\subsection{Data Collection}

Data were collected through: a) Interview by using questionnaire. Interviews were intended to collect personal identity data, characteristics and history of disease in samples; b) Physical evaluation by observing the presence of clinical signs such as aged skin, and then assess the skin brightness and elasticity using facial imaging software (Adobe Photoshop) and skin analyzer with standar present in lower arm skin. Patients were then photographed in sitting straight position with both hands extended upward and facing up. Photographs were taken from front, right, and left position. A lesion map was also created in image; c) samples were treated with the topical application of $0.25 \%$ turmeric extract. Each subject that met inclusion criteria were photographed for initial data and during the progress of the study.

\subsection{Data Analysis}

The data obtained in this study, will be processed using statistic program SPSS 21.0 with comparative tests using Willcoxon methods.

\section{Results}

An analytical observational study with prospective cohort method was conducted to compare between $0.25 \%$ turmeric extract cream and grated turmeric in skin discoloration. The study was conducted in Dr. Wahidin Sudirohusodo Makassar hospital, Hasanuddin University teaching hospital, and other networked hospitals as sample collection sites. The study was conducted during 21 days until the minimum required samples was met.

Total sample in this study who met the inclusion criteria was 22 women and then divided into two groups. First group $(n=10)$ received treatment in their upper right arm with the application of $0.25 \%$ turmeric extract cream, lower right arm with the application of $4 \% \mathrm{Hq}$ (control), upper left arm with the application of grated turmeric, and lower left arm with the application of $4 \% \mathrm{Hq}$ (control), with frequency once a day consecutively and the measured for skin discoloration at day 0,8 , and 21 by using skin analyzer. The second group (n $=12$ ) received treatment in their upper right arm with the application of $0.25 \%$ turmeric extract cream, lower right arm with the application of $4 \% \mathrm{Hq}$ (control), upper left arm with the application of grated turmeric, and lower left arm with the application of $4 \% \mathrm{Hq}$ (control), with frequency twice a day consecutively and the measured for skin discoloration at day 0,8 , and 21 by using skin analyzer. After the application of cream, no irritation, erythema, pruritus or heat sensation were observed in all subjects from the beginning of the application, during application and last day of application. According to Wilcoxon test, there was a significant difference $(\mathrm{P}<0.05)$ between $0.25 \%$ turmeric extract cream and $4 \% \mathrm{Hq}$ at day 8 (Appendix, Table 1).

The comparison of skin discoloration at day 0,8 and 21 with $0.25 \%$ turmeric extract cream and skin discoloration at day 0,8 , and 21 with grated turmeric between once application and twice application indicated that once application in $0.25 \%$ turmeric extract cream and grated cream showed no significant difference in mean score of skin discoloration at day 0,8 , and $21(\mathrm{p}>0.05)$. Mean score at day 0 increased at day 21 in $0.25 \%$ turmeric extract cream group. Mean score at day 0 increased at day 21 in grated turmeric group. For twice application in $0.25 \%$ turmeric extract cream, there was a significant mean score difference in skin discoloration with the highest score was observed at day 0 $(2.58)$ and the lowest was at day $21(1.58)(\mathrm{p}<0.05)$. In grated turmeric group, there was a significant mean difference in skin discoloration with the highest score was observed at day $0(2.58)$ and the lowest at day $21(2.25)(\mathrm{p}<0.05)$ (appendix, Table 2).

According to post hoc test between D0, D8, and D21, the $0.25 \%$ turmeric extract cream and grated turmeric with once and twice application indicated that $0.25 \%$ turmeric extract cream group with twice application at D21 - D0 and D21 D8 was significantly different $(\mathrm{P}<0.005)$ (appendix, Table 3 ).

The comparison of skin color (pigment) between $0.25 \%$ turmeric extract and grated turmeric at day $0,8,21$ with once application indicated skin discoloration at day 0,8 , and 21 which were not different significantly $(\mathrm{P}>0.005)$ (appendix, Table 4).

The comparison of skin discoloration between $0.25 \%$ turmeric extract cream and grated turmeric at day $0,8,21$ with twice application indicated that there was skin pigment discoloration at day 0,8 , and 21 which was not significantly different $(\mathrm{P}>0.005)$ (appendix, Table 5).

\section{Discussion}

This study indicated that with twice applications in the comparison between $0.25 \%$ turmeric extract and $4 \% \mathrm{Hq}$ at 
day 8 (2.30), a significant different was found $(\mathrm{P}<0.05)$ in skin pigment discoloration. In the comparison of $0.25 \%$ turmeric extract with twice application a day between D21 D0 and D21 - D8, there was a significant difference $(\mathrm{P}<0.05)$.

Become older and aged is a natural process that will be faced by all living creatures; plants, animals or human. This process is required by the nature to make sure the generation turnover and the preservation of nature content from time to time. Even though becoming older is something that must happen, but the efforts to prevent it never recede, at least to delay the aging process. It is true that there are various disturbances, either from external or internal that cause someone to become older faster (premature aging syndrome) which is usually can be prevented more easily. [9], [10].

This study involved 22 subjects with dull skin color, according to Glogan criteria. The obtained samples had youngest age of 30 years old the oldest was 45 years old. This is in accordance with literature that state that extrinsic aging has started to occur at second decade of life. From physical evaluation to establish premature aging diagnosis, all subjects were found to have dull skin color. There are four categories of photoaging according to Glogan classification, namely: I. No. Wrinkles; II. Wrinkles on Motion; III. Wrinkles at rest; IV. Only Wrinkles. [11]

The obtained data indicated that women dominated the premature aging patients. This is because one the premature aging factor is hormonal factor in women such as estrogen, progesterone, testosterone, DHEA, premenopause and menopause that can result in premature aging (Smith, 2009). The hormonal effect in women can enter the climacterium or menopause period, in which the ovary has decreased function that the produced estrogen decreases causing some manifestations including the rough skin, dull skin, and other manifestations.

In this study, the twice application in the comparison between $0.25 \%$ turmeric extract cream and $4 \% \mathrm{Hq}$ at day 8 (2.30) differed significantly $(\mathrm{P}<0.05)$ in skin discoloration (pigment). In the comparison of $0.25 \%$ turmeric extract with twice application a day between D21-D0 and D21-D8, there was significant different $(\mathrm{P}<0.05)$. Tetrahydrocurcuminoid (THC) is a free dye derived from curcuminoid, natural phenolic yellow of antioxidant compound extracted from turmeric root (Curcuma longa, family: Zingiberaceae). Curcuminoid prevents the formation of free radicals in biological systems, playing role in "bioprotectant" mechanism that protect living cells integrity. The antioxidant effect of curcuminoid combined with its inhibitory effect is known in cyclooxigenase 2 (COX-2), therefore it is useful as material in the formulation of anti-aging and in the topical formulation to maintain health and skin integrity. THC has also been found to inhibit tyrosinase acticity, an enzyme that plays role in melanogenesis process, so that can prevent the formation of melanin that can repair dryness and enlighten the skin. [7]

These findings are in accordance with Majeed et al (2001) who studied the effect and safety of $0.25 \%$ turmeric cream as a safe and natural whitening agent compared to $4 \%$ hydroquinon, so that can be used as a safe alternative to whitening agent in addition to $4 \%$ hydroquinon. That study also explained the safety and mechanism of the turmeric that can inhibit tyrosinase, making it more effective than kojic acid and ascorbic acid. [8]

The mechanism by which hydroquinone can enhance the skin is through toxic effect ot hydroquinone on melanocyte (cells in which melanin is synthesized in the skin) and through the inhibition of melanogenesis (melanin formation process). The toxic effect of hydroquinone occurs when the hydroquinone competes with tyrosine as a substrate for tyrosinase (enzyme that plays a role in melanin formation), so that the tyrosinase oxidizes hydroquinone and produce benzoquinone which is toxic to melanocyte [4].

Hydroquinone inhibits the conversion of DOPA (dihydroxy phenil alanine) into DOPAquinone in melanogenesis process, so that it inhibits the work of tyrosinase enzyme (Robert, 2009). The hydroquinone concentration varies from $2 \%$ to $5 \%$ with the high concentration is usually more iritative and has greater risk on phototoxicity [4].

Prakash \& Majeed (2009) have studied the natural antioxidant that repairs cells damage due to free radicals, an important component in anti-aging formulation. The antioxidant has the potential to prevent damage in tissues, has biochemical reaction as inflammation mediator so that it can influence the melanin synthesis by affecting the cell proliferation and melanocyte cell function and blood circulation in the skin becomes normal. Therefore, antiinflammation is incorporated into anti-aging formulation agents, that can ease, cure and protect and repair the skin integration. Polyphenol can be found in green tea, turmeric, red wine (resveratol), grapes (anthocyanin), grapes seeds (Pro anthocyanins), Indian gooseberry (galotanin), that have been scientifically proved for their efficacy. [10]

\section{Conclusion}

This study concludes that the twice application in the comparison between $0.25 \%$ turmeric extract cream and $4 \%$ $\mathrm{Hq}$ at day 8 (2.30) was significantly different in skin discoloration (pigment). In the comparison of $0.25 \%$ turmeric extract cream with twice application a day between D21-D0 and D21-D8, a significant difference was observed $(\mathrm{P}<0.05)$. There was a tendency for decreased pigment level but not significant $(\mathrm{P}<0.05)$, after the application of $0.25 \%$ turmeric extract compared to control and between grated turmeric and control at day 8 and 21 with once application a day. There was a tendency for decreased pigment level but not significant $(\mathrm{P}<0.05)$, after the application of $0.25 \%$ turmeric extract compared to control and between grated turmeric and control at day 8 and 21 with twice application a day. This study suggests further studies using higher turmeric cream level. The future studies can assess the skin change in longer period (2-3 months) and different parameters can be studied. 


\section{Appendix}

Table 1. Comparison of Skin Discoloration between 0,25\% Turmeric Extract Cream and 4\% Hydroquinone with one application.

\begin{tabular}{|c|c|c|c|c|c|c|c|c|c|c|c|c|c|}
\hline & Right Arm & & & & & & & Left Arm & & & & & \\
\hline Day & Cream & $\mathbf{n}$ & Median & Mean & SD & $\mathbf{p}$ & Day & Cream & $\mathbf{n}$ & Median & Mean & SD & $\mathbf{p}$ \\
\hline \multirow{2}{*}{0} & Turmeric Cream & 10 & 3,00 & 2,40 & 0,966 & \multirow{2}{*}{0,257} & \multirow{2}{*}{0} & Grated Turmeric & 10 & 2,00 & 2,10 & 0,876 & 0,180 \\
\hline & $\mathrm{Hq} 4 \%$ & 10 & 2,00 & 2,10 & 0,876 & & & $\mathrm{Hq} 4 \%$ & 10 & 3,00 & 2,40 & 0,843 & \\
\hline \multirow{2}{*}{8} & Turmeric Cream & 10 & 3,00 & 2,30 & 0,949 & \multirow{2}{*}{0,046} & \multirow{2}{*}{8} & Grated Turmeric & 10 & 2,250 & 2,30 & 0,823 & 0,408 \\
\hline & Hq $4 \%$ & 10 & 1,00 & 1,50 & 0,707 & & & $\mathrm{Hq} 4 \%$ & 10 & 2,00 & 2,00 & 0,816 & \\
\hline \multirow[b]{2}{*}{21} & Turmeric Cream & 10 & 2,00 & 2,10 & 0,876 & \multirow{2}{*}{0,163} & \multirow[b]{2}{*}{21} & Grated Turmeric & 10 & 2,50 & 2,30 & 0,823 & 0,096 \\
\hline & $\mathrm{Hq} 4 \%$ & 10 & 1,00 & 1,60 & 0,843 & & & $\mathrm{Hq} 4 \%$ & 10 & 2,00 & 1,80 & 0,789 & \\
\hline
\end{tabular}

Wil Cocxon test

Table 2. Comparison of Skin Discoloration at Day 0, 8, 21 with 0.25\% Turmeric Extract Cream and Skin Discoloration at Day 0, 8, 21 with Grated Skin at once and twice Application.

\begin{tabular}{|c|c|c|c|c|c|c|c|}
\hline Application & Cream & Day & $\mathbf{n}$ & Median & Mean & SD & $\mathbf{p}$ \\
\hline \multirow{6}{*}{ One } & \multirow{3}{*}{$0.25 \%$ Turmeric Extract Cream } & Pigment 0 & 10 & 2,00 & 2,40 & 0,966 & \multirow{3}{*}{0,073} \\
\hline & & Pigment 8 & 10 & 3,00 & 2,30 & 0,949 & \\
\hline & & Pigment 21 & 10 & 3,00 & 2,10 & 0,876 & \\
\hline & \multirow{3}{*}{ Grated Turmeric } & Pigment 0 & 10 & $2 d$ & 2,10 & 0,876 & \multirow{3}{*}{0,657} \\
\hline & & Pigment 8 & 10 & 3,00 & 2,30 & 0,823 & \\
\hline & & Pigment 21 & 10 & 2,00 & 2,30 & 0,823 & \\
\hline \multirow{5}{*}{ Two } & \multirow{2}{*}{$0.25 \%$ Turmeric Extract Cream } & Pigment 0 & 12 & 3,00 & 2,58 & 0,669 & \multirow{2}{*}{0,000} \\
\hline & & Pigment 21 & 12 & 2.50 & 1,58 & 0,669 & \\
\hline & \multirow{3}{*}{ Grated Turmeric } & Pigment 0 & 12 & 2,00 & 2,58 & 0,515 & \multirow{3}{*}{0,002} \\
\hline & & Pigment 8 & 12 & 3.00 & 2,33 & 0,985 & \\
\hline & & Pigment 21 & 12 & 2,00 & 2,25 & 0,754 & \\
\hline
\end{tabular}

Friedmen test

Table 3. Post Hoc Test between D0, D8, D21. 0.25\% Turmeric Extract Cream, Grated Turmeric with one and two applications.

\begin{tabular}{|c|c|c|c|c|c|}
\hline Application & Cream & & D8 - D0 & D21 - D0 & D21 - D8 \\
\hline \multirow{4}{*}{ One } & \multirow{2}{*}{ Turmeric Cream } & $\mathrm{Z}$ &,$- 272^{b}$ &,$- 828^{\mathrm{b}}$ &,$- 557^{\mathrm{b}}$ \\
\hline & & $P$ & 0,785 & 0,408 & 0,577 \\
\hline & \multirow{2}{*}{ Grated Turmeric } & $\mathrm{Z}$ &,$- 816^{\mathrm{c}}$ &,$- 513^{c}$ &, $000^{\mathrm{d}}$ \\
\hline & & $P$ & 0,414 & 0,608 & 1,000 \\
\hline \multirow{3}{*}{ Two } & \multirow{2}{*}{ Turmeric Cream } & Z & $-1,342^{b}$ & $-2,972^{b}$ & $-2,460^{b}$ \\
\hline & & $P$ &, 180 & 0,003 & 0,014 \\
\hline & Grated Turmeric & $\mathrm{Z}$ & $-1,342^{b}$ & $-1,069^{b}$ &, $000^{\mathrm{d}}$ \\
\hline
\end{tabular}

Table 4. Comparison of Skin Discoloration (pigment) Between 0,25\% Turmeric Extract Cream and Grated Turmeric at Day 0, 8, 21 for one application.

\begin{tabular}{|c|c|c|c|c|c|c|}
\hline Day & Cream & $\mathbf{n}$ & Median & Mean & SD & $\mathbf{P}$ \\
\hline \multirow{2}{*}{0} & Turmeric Cream & 10 & 3,00 & 2,40 & 966 & 0,083 \\
\hline & Grated Turmeric & 10 & 2,00 & 2,10 &, 876 & \\
\hline \multirow{2}{*}{8} & Turmeric Cream & 10 & 3,00 & 2,30 & ,949 & 1,000 \\
\hline & Grated Turmeric & 10 & 2,50 & 2,30 &, 823 & \\
\hline \multirow{2}{*}{21} & Turmeric Cream & 10 & 2,00 & 2,10 & ,876 & \\
\hline & Grated Turmeric & 10 & 2,50 & 2,30 & ,823 & 0,527 \\
\hline
\end{tabular}

Will Cocxon

Table 5. Comparison of Skin Discoloration (pigment) Between 0,25\% Turmeric Extract Cream and Grated Turmeric at Day 0, 8, 21 for two applications.

\begin{tabular}{|c|c|c|c|c|c|c|}
\hline Day & Cream & $\mathbf{n}$ & Median & Mean & SD & $\mathbf{P}$ \\
\hline \multirow{2}{*}{0} & Turmeric Cream & 12 & 3,00 & 2,58 & 669 & 1,000 \\
\hline & Grated Turmeric & 12 & 3,00 & 2,58 &, 515 & \\
\hline \multirow{2}{*}{8} & Turmeric Cream & 12 & 2,50 & 2,33 & ,778 & 1,000 \\
\hline & Grated Turmeric & 12 & 3,00 & 2,33 & ,985 & \\
\hline \multirow{2}{*}{21} & Turmeric Cream & 12 & 1,50 & 1,58 & 669 & 0,074 \\
\hline & Grated Turmeric & 12 & 2,00 & 2,25 &, 754 & \\
\hline
\end{tabular}

Will Cocxon 


\section{References}

[1] Arct J, Anna R, Mieloch M, Witulska M. (2014.) Evaluation of skin Colouring Properties of Curcuma Longa Extract. Department of Cosmetics Chemistry, Academy of cosmetics and Health Care, warsaw, podwale 13, 00-252, Poland.

[2] Bentley et al. (2008). Health Skin Reflects Your Body. P2025 .

[3] Chu et al. (2012). Development and Structure of Skin. In Goldsmith L. A., Katz, S. I; Cunningham W. Aging and Photoaging. In Textbook of Cosmetic Dermatology.

[4] Hexsel et al. (2007). Ethnic Cosideration in the Treatment of Hispanic and Latin American Patient Whith Hyperpigmentation. J Dermatol. 156 (1): 07-12.

[5] Jain et al. (2007). PHCOG MAG: Plant Review Recent Trends in Curcuma Longa Linn. Pharmacognosy Reviews. 1: 1.
[6] Majeed et al. (2010). A Randomized, Double - Blind, PlaceboControlled, Comperative Study "The Safety and Efficacy of $0,25 \%$ Tetrahydrocurcumin (Turmeric) Cream as Depigment Agent Against 4\% Hydroquinone Cream" Sabinsa Corporation USA. Philippines: Research Institute for Tropical Medicine Section of Dermatology.

[7] Prakash \& Majeed. (2009). Eurocosmetics. pp 19-23.

[8] Robert. (2009). Melasma Medical Treadment And Procedural Therapy. In: Kelly Ap, Taylor Sc., editors. Dermatology For Skin Of Colour. New York: McGraw-Hill.

[9] Septiatin. (2008). Medicinal Plant Series; Live Pharmacy from Spices, Ornamental Plants and Wild Plants. Bandung: Yrama Widya.

[10] Sjarif et al. (2012). Aged Skin. Cosmetic Dermatology. Medical Cosmetics Science Guide $2^{\text {nd }}$ Edition. Jakarta: FKUI.

[11] Smith. (2009). Comprehensive Look at Hormones and Effect of Hormone Replacement. Director of the Followiship in Anti - Aging and Functioanal Medicine Program. 